\title{
Isoimperatorin ameliorates osteoarthritis by downregulating the mammalian target of rapamycin $\mathrm{C} 1$ signaling pathway
}

\author{
JIAYAO OUYANG $^{1 *}$, HUAJI JIANG ${ }^{2 *}$, HANG FANG $^{1}$, WENBO CUI $^{3}$ and DAOZHANG CAI ${ }^{1}$ \\ ${ }^{1}$ Department of Orthopaedics, The Third Affiliated Hospital of Southern Medical University, Guangzhou, \\ Guangdong 510630; ${ }^{2}$ Department of Pain, Yue Bei People's Hospital, Shaoguan, Guangdong 512000; ${ }^{3}$ Department of \\ Orthopaedics, Dongguan Eighth People's Hospital, Dongguan, Guangdong 523326, P.R. China
}

Received November 17, 2016; Accepted June 29, 2017

DOI: $10.3892 / \mathrm{mmr} .2017 .7777$

\begin{abstract}
Osteoarthritis (OA) is the most common disease of the joints, and is characterized by the breakdown of cartilage and degradation of the extracellular matrix. OA causes a high level of patient suffering and incurs large societal costs; however, the current strategies for treating OA are restricted due to limited understanding of the underlying molecular and cellular mechanisms. In the present study, the beneficial effects of isoimperatorin (Iso) were investigated using an experimental mouse model of OA, and its mechanism of action on primary chondrocytes was elucidated. Destabilization of the medial meniscus was performed on 8-week-old male mice to induce OA in the knees. Iso $(500 \mathrm{mg} / \mathrm{g} /$ day $)$ was intragastrically administered for 4 weeks. Degeneration of articular cartilage was assessed by histology using the Osteoarthritis Research Society International scoring system. The expression of matrix metalloproteinase (MMP)13, Runt-related transcription factor (Runx)2, type X collagen ( $\mathrm{Col} \mathrm{X}$ ) and
\end{abstract}

Correspondence to: Dr Daozhang Cai, Department of Orthopaedics, The Third Affiliated Hospital of Southern Medical University, 183 West Zhongshan Avenue, Guangzhou, Guangdong 510630, P.R. China

E-mail: daozhang@medmail.com.cn

Dr Wenbo Cui, Department of Orthopaedics, Dongguan Eighth People's Hospital, 68 Dongguan Southern Road of West Lake, Dongguan, Guangdong 523326, P.R. China

E-mail: ji617409@163.com

*Contributed equally

Abbreviations: OA, osteoarthritis; DMM, destabilization of the medial meniscus; MMP, matrix metalloproteinase; Runx2, Runt-related transcription factor 2; Col X, type X collagen; VEGF, vascular endothelial growth factor; Iso, isoimperatorin; COX-2, cyclooxygenase-2; TAC, thickness of articular cartilage; OARSI, Osteoarthritis Research Society International; mTORC1, mammalian target of rapamycin complex 1

Key words: isoimperatorin, osteoarthritis, hypertrophic chondrocyte, angiogenesis, autophagy, mammalian target of rapamycin vascular endothelial growth factor (VEGF) in the knee joints was examined by immunohistochemistry. In vitro, murine primary chondrocytes were treated with various concentrations of Iso, followed by $10 \mathrm{ng} / \mathrm{ml}$ interleukin- 1 . The mRNA expression levels of MMP13, Runx2, Col X and VEGF were determined by reverse transcription-quantitative polymerase chain reaction. The levels of autophagy and mammalian target of rapamycin (mTOR) signaling were determined by western blotting. Iso significantly ameliorated the severity of articular cartilage degradation in mice with experimental OA. The expression levels of MMP13, Runx2, Col X and VEGF were reduced in Iso-treated mice. In murine primary chondrocytes, Iso also reduced MMP13, Runx2, Col X and VEGF expression, and activated autophagy by downregulating the mTOR complex 1 (mTORC1) signaling pathway. Therefore, the results of the present study demonstrated that Iso ameliorates OA-induced pathological alterations by delaying chondrocyte deterioration, activating autophagy and inhibiting mTORC1, which suggests that Iso may have therapeutic potential for attenuating articular cartilage degradation and treating OA.

\section{Introduction}

Osteoarthritis (OA) is the most common musculoskeletal disease worldwide, which primarily affects the knees, hands and feet, leading to a reduction in patient quality of life. The incidence of symptomatic knee OA is highest amongst individuals aged between 55 and 64 years, and the prevalence increases with age. It has been reported that $\sim 9.29 \%$ of the US population is diagnosed with symptomatic knee OA by the age of 60 (1). The development of OA is associated with various risk factors including genetic factors, an epigenetic predisposition, aging, being female, injury, mechanical stress, diet and lifestyle (2). The natural process of aging is associated with $\mathrm{OA}$ and the key chondrocyte senescence markers, including senescent-associated- $\beta$-galactosidase, senescence-associated heterochromatin foci, p53, p16, caveolin 1 and telomere length (3). The progression of OA is characterized by chondrocyte deterioration, degradation of the extracellular matrix, subchondral bone remodeling, formation of ectopic bone and synovitis. Under normal conditions, a balance between matrix proteins and matrix-degrading enzymes is maintained in chondrocytes (4). However, a disruption in the normal resting 
state of chondrocytes may lead to matrix remodeling, inappropriate hypertrophic maturation and cartilage calcification. A previous study demonstrated that interleukin (IL)-1 stimulates the synthesis and activity of matrix metalloproteinases (MMPs), as well as other enzymes involved in OA-induced cartilage destruction (5). Hypertrophic chondrocytes produce catabolic proteins, including MMP13, Runt-related transcription factor (Runx) 2 and type X collagen (Col X) (6). High levels of vascular endothelial growth factor (VEGF) are also observed in patients with OA, which implies that angiogenesis may contribute to the pathogenesis of OA (7). Previous studies have investigated novel drugs to repair cartilage lesions, such as $\beta$-defensin- 4 , which is thought to be a protected marker in the repair process of OA $(8,9)$. In clinical practice, numerous drugs, including nonsteroidal anti-inflammatory drugs, cyclooxygenase-2 (COX-2) inhibitors (10), glucosamine, steroids and hyaluronan (11) have been used to delay OA in patients. However, these drugs do not reverse the effects of OA. Therefore, patients with OA must be treated with arthroplasty, which is associated with a large economic burden. Therefore, it is necessary to identify a novel agent for the treatment of OA.

Isoimperatorin [Iso; 4-(3-methyl-2-butenyl)-7H-furo (3,2-g) benzopyran-7-one] is a linear furanocoumarin and one of the main components of Prangos ferulacea (12). Iso is a potent medicinal herbal compound that is also isolated from the roots of Angelicae dahurica (13). A. dahurica suppresses inducible nitric oxide synthase (iNOS) and nitric oxide production (14). In addition, as an extract from A. dahuricae, Iso has been used as a vasodilatory, antiallergic and anticancer drug in clinical practice (15-17). Notably, Iso also serves an active anti-inflammatory role, and can inhibit COX-2 and prostaglandin E2 production in mouse models $(18,19)$. A previous study revealed that Iso can reduce the production of reactive oxygen species via tumor necrosis factor- $\alpha$-induced AKT, extracellular signal-regulated kinase and protein kinase $\mathrm{C}$ signaling in human cells $(16,20)$. However, whether Iso possesses chondroprotective properties remains to be elucidated. The present study aimed to establish the effects of Iso in delaying chondrocyte deterioration in OA in vivo and in vitro, and to determine the potential underlying mechanisms for novel treatments of OA. The results of the present study demonstrated that Iso delayed the progression of OA in vivo and in vitro. In addition, autophagy activation and mammalian target or rapamycin complex 1 (mTORC1) inhibition were responsible for the effects of Iso administration on OA.

\section{Materials and methods}

Animal model and treatments. C57/BL6 mice (age, 8 weeks; male; weight, 18-25 g) were purchased from the Laboratory Animal Centre of the Southern Medical University (Guangzhou, China). The mice had free access to standard chow and water; the mice were maintained at an optimal temperature $\left(24-26^{\circ} \mathrm{C}\right)$ and at $70 \%$ humidity under a $12-\mathrm{h}$ light/dark cycle. The mice were anesthetized with $8 \mu \mathrm{l} / \mathrm{g}$ (anesthetic volume/mice weight) of $1 \%$ Amobarbital sodium prior to surgery. The OA model was established by selectively transecting the medial meniscus (destabilization of the medial meniscus; DMM). Mice ( $\mathrm{n}=15)$ were randomly divided into the following three groups: Sham, OA and OA + Iso (n=5/group).
In the two surgical groups (OA and OA + Iso), the right medial collateral ligaments were dissected, which was followed by DMM to induce the OA model. In the sham-operated group, only the skin of the right knee joint was resected. Following the induction of $\mathrm{OA}$, all mice in the $\mathrm{OA}+$ Iso group were treated with Iso (Sigma-Aldrich; Merck KGaA, Darmstadt, Germany). Iso was delivered intragastrically via oral administration $(500 \mathrm{mg} / \mathrm{g} / \mathrm{day})$ using syringe feeding. At 4 weeks post-surgery, mice from each group were sacrificed and the right knee joints were collected. Animal experiments were approved by the Animal Experimental Ethics Committee of Southern Medical University (Guangdong, China).

\section{Systematic assessment of $O A$}

Histological staining and OA scoring. The right knee joints were isolated and all tissues were fixed with $4 \%$ paraformaldehyde in $0.1 \mathrm{M}$ phosphate-buffered saline overnight at $4^{\circ} \mathrm{C}$. They were then decalcified with $14.5 \%$ EDTA ( $\mathrm{pH} \mathrm{7.4)}$ at room temperature for $\sim 20$ days. Following decalcification, the specimens were routinely embedded in paraffin, cut into $5-\mu \mathrm{m}$ sections along the sagittal plane and stained with Safranin O/Fast Green and toluidine blue at room temperature for $15 \mathrm{~min}$. Under an Olympus light microscope (magnification, x200; Olympus Corporation, Tokyo, Japan) three fields of tibial articular cartilage were randomly selected and the number of chondrocytes was calculated to obtain a mean value. OA was evaluated using the Osteoarthritis Research Society International (OARSI) semi-quantitative scoring system, in order to verify that cartilage pathology varied in severity by location (21). In this system, the scores are defined as follows: 0 , normal cartilage; 0.5 , loss of proteoglycan with an intact surface; 1 , superficial fibrillation without loss of cartilage; 2, vertical clefts and loss of surface lamina; 3, vertical clefts/erosion to the calcified layer lesion in $1-25 \%$ of the quadrant width; 4 , lesion reaches the calcified cartilage in $25-50 \%$ of the quadrant width; 5 , lesion reaches the calcified cartilage in $50-75 \%$ of the quadrant width; 6 , lesion reaches the calcified cartilage in $>75 \%$ of the quadrant width. Three different areas from the tibial plateau were randomly selected to calculate and evaluate the OARSI scores. The average thickness of the articular cartilage (TAC) in the tibial plateau was measured using Image-Pro Plus version 6.0 software (Media Cybernetics, Inc., Rockville, MD, USA).

Immunohistochemistry. For immunohistochemical analysis, endogenous peroxidase in deparaffinized sections (3-5 $\mu \mathrm{m})$ was blocked via incubation with $3 \%$ hydrogen peroxide at room temperature for $10 \mathrm{~min}$. The sections were then incubated with rabbit anti-MMP13 (1:150; cat. no. A1606; ABclonal Biotech Co., Ltd., Woburn, MA, USA), anti-Runx2 (1:75; cat. no. A2851; ABclonal Biotech Co., Ltd.), anti-Col X (1:75; cat. no. ab58632; Abcam, Cambridge, UK) and anti-VEGF (1:100; cat. no. A5708; ABclonal Biotech Co., Ltd.) at $4^{\circ} \mathrm{C}$ overnight. Subsequently, sections were incubated with anti-rabbit secondary antibody (1:100; cat. no. RM3002; Beijing Ray Antibody Biotech, Beijing, China) at $37^{\circ} \mathrm{C}$ for $\sim 1 \mathrm{~h}$. Immunohistochemistry was performed to detect MMP13, Runx2, Col X and VEGF using a diaminobenzidine tetrahydrochloride kit (cat. no. ZLI-9017; OriGene Technologies, Inc., Beijing, China) for $1 \mathrm{~min}$ followed by counterstaining with 
Table I. Primer sequences for reverse transcription-quantitative polymerase chain reaction.

\begin{tabular}{llll}
\hline Gene ID & Gene & \multicolumn{1}{c}{ Forward (5'-3') } & Reverse $\left(5^{\prime}-3^{\prime}\right)$ \\
\hline NM_008607.2 & MMP13 & CTTCTTCTTGTTGAGCTGGACTC & CTGTGGAGGTCACTGTAGACT \\
NM_001145920.2 & Runx2 & AAATTAACGCCAGTCGGAGC & CCACTTCTCGGTCTGACGAC \\
NM_009925.4 & Col X & TTCTGCTGCTAATGTTCTTGACC & GGGATGAAGTATTGTGTCTTGGG \\
NM_001025250.3 & VEGF & CTGCCGTCCGATTGAGACC & CCCCTCCTTGTACCACTGTC \\
NM_001289726.1 & GAPDH & AGGTCGGTGTGAACGGATTTG & TGTAGACCATGTAGTTGAGGTCA
\end{tabular}

Col X, type X collagen; MMP, matrix metalloproteinase; Runx2, Runt-related transcription factor; VEGF, vascular endothelial growth factor.

hematoxylin for $15 \mathrm{sec}$ at room temperature. The percentage of positive cells in three random fields among the total area was calculated to obtain a mean value using an Olympus light microscope (light, magnification, x200; Olympus Corporation).

Isolation and culture of murine primary chondrocytes. Chondrocytes were isolated from the knees of 4-day-old C57/BL6 male mice (Laboratory Animal Centre of the Southern Medical University) via digestion at $37^{\circ} \mathrm{C}$ with $0.25 \%$ trypsin-EDTA for $30 \mathrm{~min}$ and then $0.25 \%$ collagenase II for $6 \mathrm{~h}$. The cells were suspended following centrifugation at $1,000 \mathrm{x} \mathrm{g}$ for $5 \mathrm{~min}$ at room temperature in Dulbecco's modified Eagle's medium-F12 (cat. no. 11320-033; Gibco; Thermo Fisher Scientific, Inc., Waltham, MA, USA) supplemented with 5\% fetal bovine serum (Thermo Fisher Scientific, Inc.), $100 \mathrm{U} / \mathrm{ml}$ penicillin and $100 \mu \mathrm{g} / \mathrm{ml}$ streptomycin (Life Technologies; Thermo Fisher Scientific, Inc.), and then cultured at $37^{\circ} \mathrm{C}$ in an atmosphere containing $5 \% \mathrm{CO}_{2}$ and $95 \%$ humidity.

Cell viability assay. The cytotoxicity of Iso on chondrocytes was assessed using a Cell Counting kit-8 (CCK8) assay (cat. no. KGA317; Nanjing KeyGen Biotech Co., Ltd., Nanjing, China). The cells were seeded onto 96 -well culture plates at $1 \times 10^{5}$ cells/well and allowed to adhere overnight at $37^{\circ} \mathrm{C}$. Following incubation, cells were treated with 1-100 mM Iso at $37^{\circ} \mathrm{C}$ for $24 \mathrm{~h}$, the culture medium was then removed and $10 \mu \mathrm{g} / 100 \mu \mathrm{l}$ CCK8 was added to each well for incubation at $37^{\circ} \mathrm{C}$ for $4 \mathrm{~h}$. The optical density was measured at $450 \mathrm{~nm}$ using a microplate reader.

$R N A$ extraction and reverse transcription-quantitative polymerase chain reaction ( $R T-q P C R)$. The primary chondrocytes were seeded onto 96 -well culture plates at $1 \times 10^{5}$ cells/well and allowed to adhere overnight at $37^{\circ} \mathrm{C}$. Following $48 \mathrm{~h}$, the cells were serum starved overnight then treated with 1-50 $\mu \mathrm{M}$ Iso for $1 \mathrm{~h}$, prior to stimulation with $10 \mathrm{ng} / \mathrm{ml} \mathrm{IL-1} \mathrm{(cat.} \mathrm{no.} \mathrm{211-11B;}$ Peprotech, Inc., Rocky Hill, NJ, USA) at room temperature for $24 \mathrm{~h}$. Following $24 \mathrm{~h}$, cells were collected for RT-qPCR and western blotting. RNA was extracted from monolayer cultures of chondrocytes using TRIzol reagent (Thermo Fisher Scientific, Inc.). Total RNA was isolated with methylene trichloride and isopropanol, then reversed transcribed into cDNA using the PrimeScript ${ }^{\mathrm{TM}}$ RT Reagent kit (cat. no. RR037A; Takara Bio, Inc., Otsu, Japan), according to the manufacturer's protocol. RT-qPCR was performed in triplicate using the ChamQ ${ }^{\mathrm{TM}}$ SYBR qPCR Master Mix (cat. no. Q311; Vazyme, Piscataway, NJ, USA). The cycle parameters of RT were as follows: $10 \mathrm{~min}$ at $30^{\circ} \mathrm{C}, 50 \mathrm{~min}$ for $42^{\circ} \mathrm{C}$, and $95^{\circ} \mathrm{C}$ at $5 \mathrm{~min}$. In addition, the following qPCR thermocycling conditions were used: 1 cycle at $95^{\circ} \mathrm{C}$ for $10 \mathrm{~min}$, followed by 40 cycles of $15 \mathrm{sec}$ at $95^{\circ} \mathrm{C}, 1 \mathrm{~min}$ at $60^{\circ} \mathrm{C}$ and $15 \mathrm{sec}$ at $72^{\circ} \mathrm{C}$. A melting curve was generated to verify gene-specific amplification. The primers were designed using Primer3-Blast online software (https://blast.ncbi.nlm.nih.gov/Blast.cgi). The primer pairs used for the present study are presented in Table I. Relative gene expression levels were calculated using the $2^{-\Delta \Delta \mathrm{Cq}}$ method (22). Target mRNA levels were normalized to the reference gene GADPH (Table I).

Cartilage protein extraction and western blot analysis. Western blot analyses were conducted on the protein of lysates from cultured chondrocytes, which were treated as described prior to RT-qPCR. The cells were treated with $1 \mathrm{M}$ Tris-HCl (pH 6.8) buffer containing 10\% SDS (Sigma-Aldrich; Merck $\mathrm{KGaA}$ ) and $20 \%$ glycerol for $10 \mathrm{~min}$ at $97^{\circ} \mathrm{C}$. The cell lysates were centrifuged at $1,000 \mathrm{x} \mathrm{g}$ for $5 \mathrm{~min}$ at room temperature and the supernatants were stored at $-20^{\circ} \mathrm{C}$. For electrophoresis, the concentration of the supernatants was measured using a bicinchoninic acid assay; $100 \mu \mathrm{g}$ supernatants were separated by $10 \%$ SDS-PAGE. The proteins were transferred onto nitrocellulose membranes, blocked with 5\% non-fat milk containing $0.02 \%$ Tween at room temperature for $\sim 1 \mathrm{~h}$, and incubated overnight at $4^{\circ} \mathrm{C}$ with anti- $\beta$-actin (cat. no. RM2001; 1:2,000; Beijing Ray Antibody Biotech), anti-Beclin1 (cat. no. 3495; 1:2,000; Cell Signaling Technology, Inc., Danvers, MA, USA), anti-p62 (cat. no. 5114; 1:2,000; Cell Signaling Technology, Inc.), anti-S6 kinase (S6K; cat. no. 9202; 1:3,000; Cell Signaling Technology, Inc.), anti-phosphorylated (p)-S6K (cat. no. 9204; 1:3,000; Cell Signaling Technology, Inc.), anti-eukaryotic translation initiation factor $4 \mathrm{E} /$ binding protein 1 (4E/BP1; cat. no. 9452; 1:3,000; Cell Signaling Technology, Inc.) and anti-P-4E/BP1 (cat. no. 2855; 1:3,000; Cell Signaling Technology, Inc.). The blots were then probed with horseradish peroxidase-conjugated secondary antibodies (cat. no. RM3002; 1:5,000; Beijing Ray Antibody Biotech) at room temperature for $1 \mathrm{~h}$. The antigen-antibody complexes were visualized with an Enhanced Chemiluminescence Plus detection kit (PerkinElmer, Inc., Waltham, MA, USA).

Statistical analysis. Data are presented as the mean \pm standard deviation. One-way analysis of variance followed by a post hoc 
Table II. OARSI scoring and TAC of the tibial plateau in different groups.

\begin{tabular}{lcc}
\hline Group & OARSI score & TAC $(\mu \mathrm{m})$ \\
\hline Sham & $0.30 \pm 0.27^{\mathrm{a}}$ & $127.62 \pm 13.36^{\mathrm{a}}$ \\
OA & $6.80 \pm 0.84$ & $82.53 \pm 7.90$ \\
OA + Iso & $3.50 \pm 1.00^{\mathrm{a}}$ & $121.16 \pm 4.97^{\mathrm{a}}$ \\
\hline
\end{tabular}

Data are presented as the mean \pm standard deviation ( $\mathrm{n}=5$ mice/roup). ${ }^{\mathrm{a}} \mathrm{P}<0.05$ vs. OA. Iso, isoimperatorin; OA, osteoarthritis; OARSI, Osteoarthritis Research Society International; TAC, thickness of articular cartilage.

Tukey test was used to determine statistical differences. All experiments were repeated at least three times, and representative experiments are shown. Data analyses were performed using SPSS version 19.0 software (IBM Corp., Armonk, $\mathrm{NY}$, USA). $\mathrm{P}<0.05$ was considered to indicate a statistically significant difference.

\section{Results}

Iso treatment delays the progression of experimental OA. To confirm the effects of Iso on delaying OA progression, Safranin $\mathrm{O} /$ Fast Green staining was performed and the OARSI scoring system was used to evaluate the severity of OA. The results revealed that there were more fibrillations on the surface of the articular cartilage in the OA group than in the OA + Iso group. In addition, in the OA group, less chondrocytes were observed than in the OA + Iso group (Fig. 1A). TAC was assessed by toluidine blue staining (Fig. 1B). TAC was reduced in the OA group when compared with the OA + Iso group. The histological OARSI score in the tibial plateau was increased in the OA group when compared with the Sham group; however, it was significantly decreased in the OA + Iso group compared

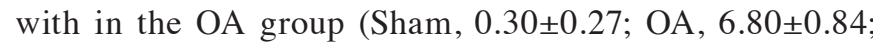
$\mathrm{OA}+$ Iso, $3.50 \pm 1.0$; $\mathrm{P}<0.05$; Fig. 1C; Table II). These results indicated that Iso treatment ameliorated degradation of articular cartilage, as verified in Fig. 1D. Cartilage thickness was significantly decreased in the OA group compared with in the Sham group; however, thickness was significantly increased in the OA + Iso group compared with in the OA group (Sham,

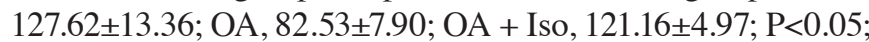
Fig. 1D; Table II).

Iso reduces the expression of MMP13, Runx2, Col X and VEGF in experimental $O A$. To investigate the mechanism underlying the protective effects of Iso on cartilage, chondrocyte hypertrophy and angiogenesis were evaluated. The expression levels of MMP13, Runx2, Col X (all hypertrophic chondrocyte markers) and VEGF (an angiogenesis marker) were detected by immunohistochemistry (Fig. 2). The percentage of MMP13-positive cells in the articular cartilage was significantly higher in the OA group compared with in the Sham group; however, it was lower in the OA + Iso group compared with in the OA group (Sham, 7.76 $\pm 5.66 \%$; OA, 50.40 $\pm 5.46 \%$; $\mathrm{OA}+$ Iso, 21.60 $\pm 2.25 \%$; $\mathrm{P}<0.05$; Fig. 2). A similar result was
A
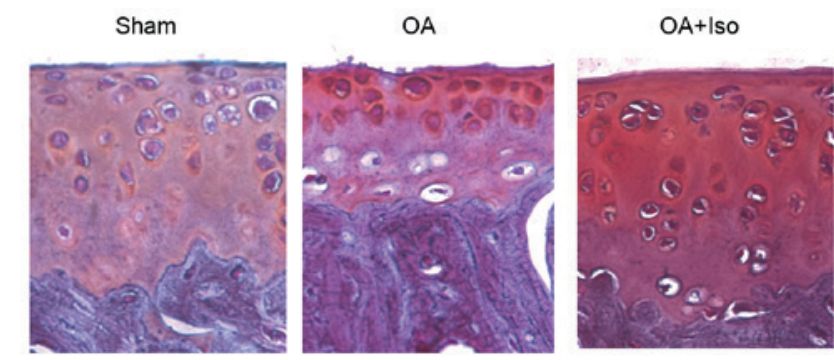

B
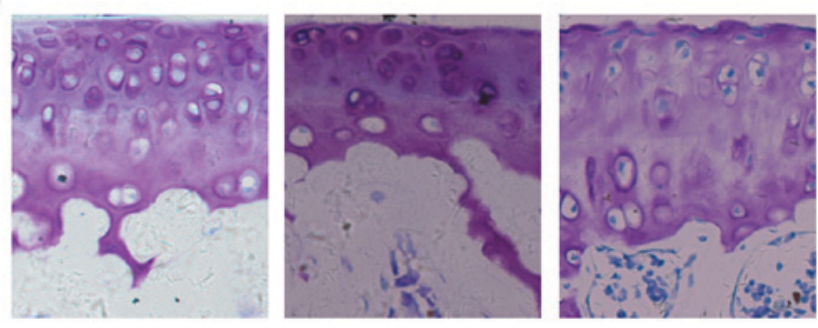

C
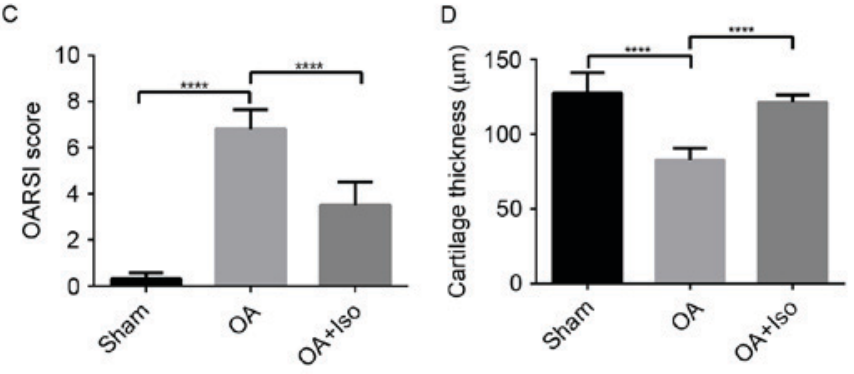

Figure 1. Histological staining and assessment following the induction of OA. (A) Safranin O-fast green staining (magnification, x200) on the tibial plateau at 4 weeks post-surgery. (B) Toluidine blue staining (magnification, $\mathrm{x} 200$ ) on the tibial plateau at 4 weeks post-surgery. (C) Histological OA grade based on the OARSI scoring system. Histological evaluation revealed a significant decrease in OA following Iso treatment. (D) Thickness of the cartilage of the tibial plateau. Data are presented as the mean \pm standard deviation ( $\mathrm{n}=5 \mathrm{mice} /$ group). ${ }^{* * * *} \mathrm{P}<0.001$ vs. OA group. Iso, isoimperatorin; OA, osteoarthritis; OARSI, Osteoarthritis Research Society International.

obtained for the percentage of Runx2-positive cells (Sham,

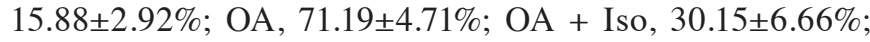
$\mathrm{P}<0.05$; Fig. 2) and Col X-positive cells (Sham, 10.94 $\pm 3.34 \%$; OA, 61.65 $\pm 6.47 \%$; OA + Iso, 20.72 $\pm 3.19 \%$; P<0.05; Fig. 2), as the percentage in the articular cartilage was significantly higher in the OA group compared with in the Sham group; however, it was significantly lower in the OA + Iso group compared with in the OA group. These results indicated that Iso reduced hypertrophic alterations in articular cartilage in the experimental OA model. In addition, the percentage of VEGF-positive cells in the articular cartilage was significantly higher in the OA group compared with in the Sham group; however, it was significantly lower in the OA + Iso group compared with in the OA group (Sham, 13.70 $\pm 3.08 \%$; OA, $74.82 \pm 9.18 \%$; OA + Iso, $14.43 \pm 3.98 \%$; P<0.05; Fig. 2). These results suggested that Iso treatment may delay the degradation of articular cartilage, in part, through the inhibition of angiogenesis.

Effects of Iso on the viability of primary chondrocytes. The potential cytotoxicity of Iso on mouse primary chondrocytes was evaluated using CCK8 assay. Iso, at concentrations 

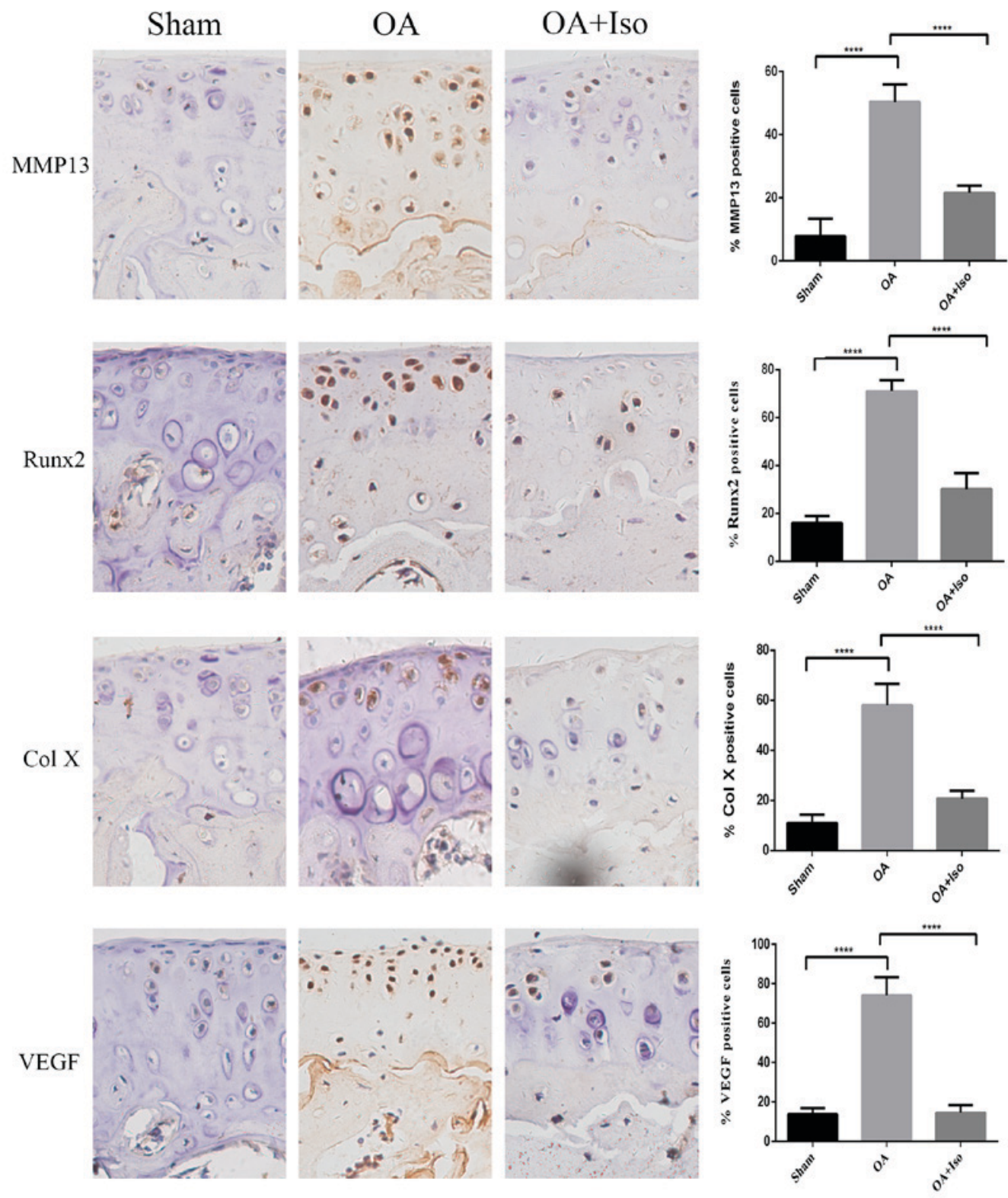

Figure 2. Immunohistochemical analysis (magnification, $\mathrm{x} 200$ ) revealed that the percentage of cells positive for MMP13, Runx2, Col X and VEGF expression was decreased in the articular cartilage of the OA + Iso group compared with the OA group. Data are presented as the mean \pm standard deviation $(\mathrm{n}=5$ mice/group). ${ }^{* * * *} \mathrm{P}<0.001$ vs. OA group. Col X, type X collagen; Iso, isoimperatorin; MMP, matrix metalloproteinase; OA, osteoarthritis; Runx2, Runt-related transcription factor; VEGF, vascular endothelial growth factor.

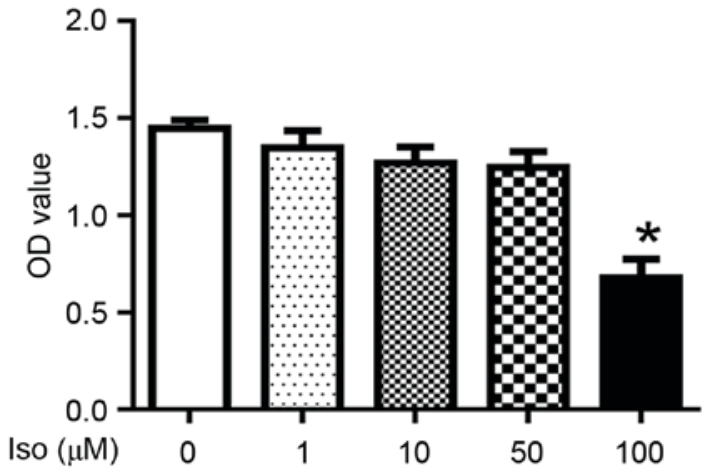

Figure 3. Effects of Iso on the cell viability of primary chondrocytes. Cells were cultured with various concentrations of Iso $(0-100 \mu \mathrm{M})$ for $24 \mathrm{~h}$. Cell viability was then determined using a Cell Counting Kit- 8 assay. Data are presented as the mean \pm standard deviation of three experiments. ${ }^{*} \mathrm{P}<0.05$ vs. $0 \mu \mathrm{M}$ Iso. Iso, isoimperatorin; $\mathrm{OA}$, osteoarthritis; $\mathrm{OD}$, optical density. between 1 and $50 \mu \mathrm{M}$, exhibited no toxicity in chondrocytes; however, $100 \mu \mathrm{M}$ Iso significantly reduced the viability of chondrocytes ( $\mathrm{P}<0.05$; Fig. 3). Therefore, 1,10 and $50 \mu \mathrm{M}$ Iso were used in the subsequent experiments.

Iso treatment reduces MMP13, Runx2, Col X and VEGF expression in primary chondrocytes. The effects of various concentrations of Iso on murine primary chondrocytes treated with $10 \mathrm{ng} / \mathrm{ml}$ IL-1 were determined. IL-1 significantly upregulated the expression of MMP13 (Fig. 4A), Runx2 (Fig. 4B), Col X (Fig. 4C) and VEGF (Fig. 4D). However, Iso inhibited the effects of IL-1, decreasing the expression of MMP13, Runx2, Col X (chondrocyte hypertrophy) and VEGF (angiogenesis). These results demonstrated that Iso may inhibit chondrocyte hypertrophy and angiogenesis in vivo and in vitro. 

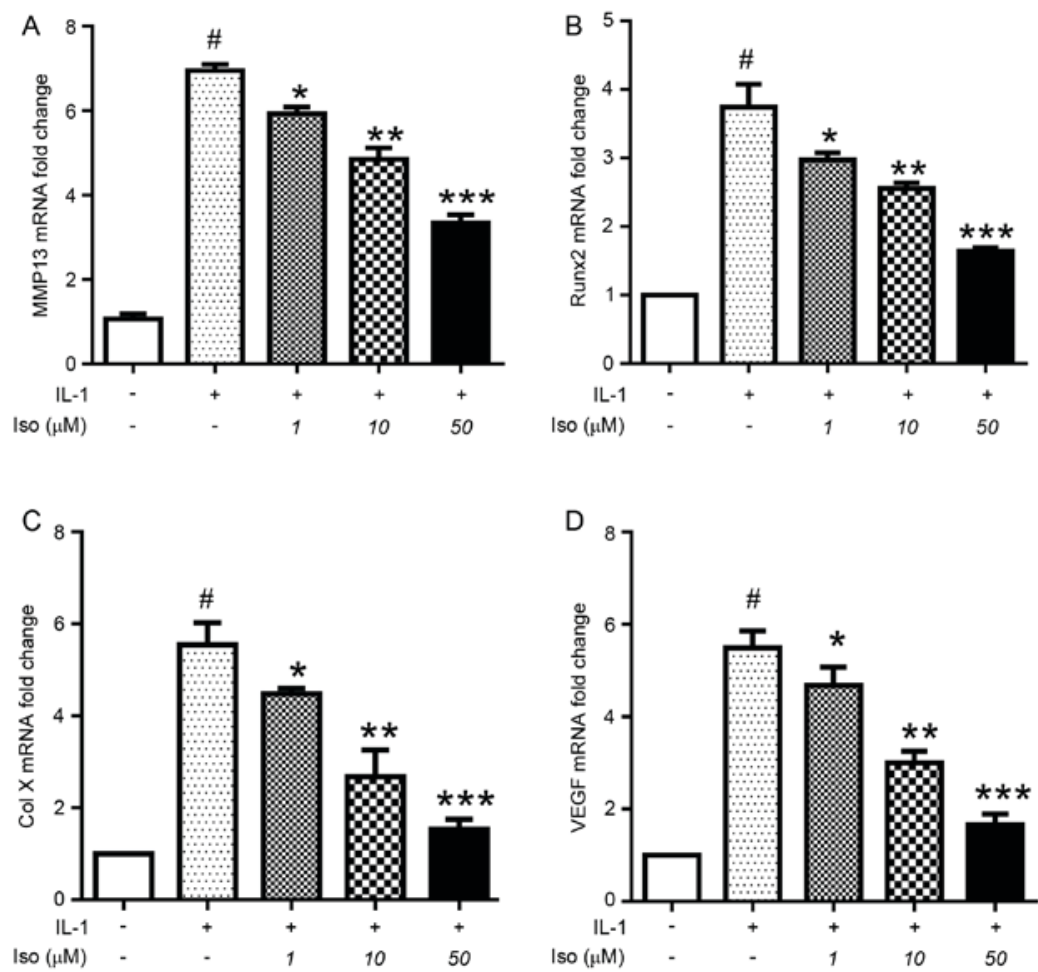

Figure 4. Iso treatment induces a reduction in the mRNA expression levels of MMP13, Runx2, Col X and VEGF in murine primary chondrocytes. Chondrocytes were pretreated with various concentrations of Iso $(0,1,10$ or $50 \mu \mathrm{M})$ for $1 \mathrm{~h}$, followed by stimulation with IL-1 (10 ng/ml) for $24 \mathrm{~h}$. The chondrocytes were collected for gene expression analysis by reverse transcription-quantitative polymerase chain reaction. Iso inhibited the mRNA expression levels of (A) MMP13, (B) Runx2, (C) Col X and (D) VEGF in a dose-dependent manner. Data are presented as the mean \pm standard deviation of three experiments.

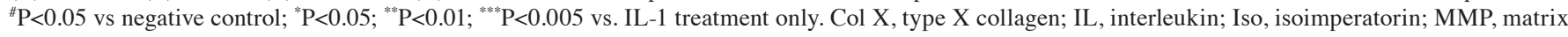
metalloproteinase; Runx2, Runt-related transcription factor; VEGF, vascular endothelial growth factor.

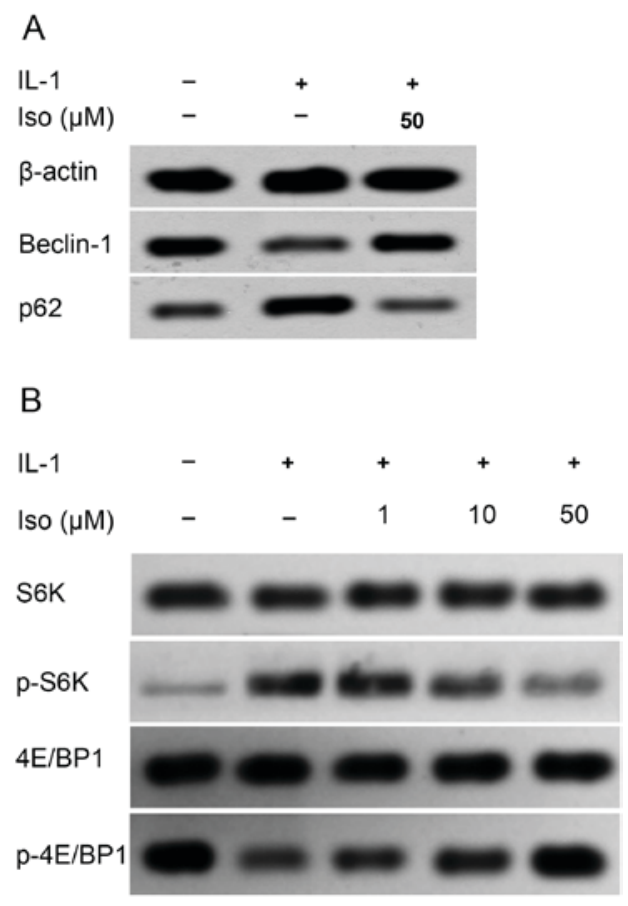

Figure 5. Iso activated autophagy and inhibited IL-1-induced mTORC1 activation. (A) Iso increased the expression of Beclin 1 and inhibited that of p62. (B) IL-1 activated mTORC1 signaling by increasing the expression of $\mathrm{p}-\mathrm{S} 6 \mathrm{~K}$ and reducing the expression of $\mathrm{P}-4 \mathrm{E} / \mathrm{BP} 1$. However, treatment with Iso reversed the effect of IL-1 in a dose-dependent manner. 4E/BP1, eukaryotic translation initiation factor $4 \mathrm{E} /$ binding protein 1 ; Iso, isoimperatorin; IL, interleukin; mTORC1, mammalian target of rapamycin complex 1; p-, phosphorylated; S6K, S6 kinase.
Iso activates autophagy signaling by enhancing expression of Beclin 1 and inhibiting that of p62. Recent evidence suggests that autophagy may be involved in the progression of OA (23); therefore, the present study examined whether Iso attenuated OA by modulating autophagy signaling. Autophagy was inhibited following IL-1 stimulation, as indicated by the reduced expression of Beclin 1 and increased expression of p62. However, Iso treatment inhibited the effects of IL-1 by enhancing the expression of Beclin 1 and reducing that of p62, indicating that Iso may attenuate OA by activating autophagy (Fig. 5A).

Disease attenuation following Iso treatment may be partly attributable to inhibition of the mTORC1 signaling pathway. The mTORC1 signaling pathway is a well-known key regulator of inflammation and angiogenesis in OA $(24,25)$. To investigate the potential mechanism through which Iso exerts its anti-OA effect, the phosphorylation status of its two direct downstream targets in the mTORC1 signaling pathway, S6K and 4E-BP1 (26), were determined by western blot analysis. Iso decreased the phosphorylation of S6K and increased 4E/BP1 phosphorylation in a dose-dependent manner, which suggested that the mTORC1 pathway was inactivated by Iso (Fig. 5B).

\section{Discussion}

Iso is a traditional Chinese herb with anti-inflammatory properties that is widely used in China. In the present study, the 
anti-OA properties of Iso were evaluated in vivo and in vitro. Iso was revealed to prevent articular cartilage degeneration by preventing chondrocyte hypertrophy and aberrant angiogenesis via MMP13, Runx2, Col X and VEGF expression, and activating autophagy by downregulating the mTORC1 signaling pathway in a dose-dependent manner. These findings were consistent in both of the models investigated: The DMM-induced OA mouse model and in IL-1-treated murine chondrocytes.

OA is a chronic degenerative process that leads to chondrocyte hypertrophy and cartilage damage (27). In OA, the rupture of cartilage homeostasis causes a subset of factors to promote cartilage damage, which leads to matrix remodeling, cartilage calcification and hypertrophy-like changes, and aggravates the progression of OA $(6,21,22,28)$. Articular cartilage refers to permanent cartilage that does not undergo terminal differentiation under normal conditions. However, when stimulated, chondrocyte differentiation leads to hypertrophy and apoptosis (28). MMP13, Runx2 and Col X are produced by hypertrophic chondrocytes and are important standard markers for chondrocyte hypertrophy $(16,18,21)$. Runx 2 is a major transcription factor involved in chondrocyte differentiation and is overexpressed in OA cartilage, elevating the production of Col X and MMP13 (28-30). Angiogenesis is also thought to affect the function and homeostasis of cartilage in the pathogenesis of OA (31). VEGF is a secreted mitogen associated with angiogenesis that has been reported to regulate the vascular invasion of the hypertrophic cartilage in OA; one isoform of which, VEGF-A, promotes subsequent cartilage vascularization $(32,33)$. A meta-analysis revealed that VEGF is upregulated in patients with OA (7) and that it contributes to the severity of knee OA $(34,35)$. Intravenous and intra-articular administration of the anti-VEGF antibody bevacizumab facilitates articular cartilage repair and attenuates the severity of OA $(36,37)$. Therefore, it is hypothesized that Iso may ameliorate OA by reducing MMP13, Runx2, Col $\mathrm{X}$ and VEGF production.

In the present study, the mechanism underlying the action of Iso on primary chondrocytes was also evaluated. Numerous signaling pathways are involved in cartilage damage in OA. Among these, the present study focused on the autophagy-associated mTORC1 signaling pathway due to its vital function in the progression of OA. Autophagy is an essential cellular homeostatic process for cartilage survival that protects articular cartilage. Previous studies have demonstrated that the genetic ablation of autophagy protein 5 in chondrocytes accelerates age-associated OA $(34,38)$. Autophagy is decreased in OA; however, the administration of rapamycin, a specific inhibitor of mTOR signaling, reverses this effect $(39,40)$. Autophagy is involved in numerous pathological processes, including anti-inflammatory, anti-hypertrophic and anti-angiogenesis activities. It is also involved in chondrocyte hypertrophy during cartilage degradation and counteracts degenerative alterations, including cartilage hypertrophy and the terminal differentiation of chondrocytes $(41,42)$. Peroxisome proliferator-activated receptor (PPAR) $\gamma$ deficiency leads to increased cartilage degradation and the aberrant production of inflammatory (iNOS and COX-2) and hypertrophic (MMP13) markers associated with upregulated mTOR signaling and autophagy inactivation. This results in increased inflammatory and hypertrophic activity in the articular cartilage and OA (24). mTOR is associated with angiogenesis, and the administration of rapamycin decreases VEGF production (25) and disrupts angiogenesis (43). mTOR interacts with numerous aspects of different cellular processes, including survival, growth and metabolism $(40-42,44,45)$. An increasing body of evidence has indicated that mTOR signaling regulates the modulation of autophagy. mTOR suppression can activate autophagy, leading to a reduction in the severity of OA $(46,47)$. Intra-articular administration of rapamycin also reduces the severity of articular cartilage degradation by reducing mTOR signaling $(48,49)$. Enhancement of the synthesis of endogenous n-3 polyunsaturated fatty acids (PUFAs) from n-6 PUFAs attenuates OA through the inhibition of mTORC1 and the promotion of autophagy in cartilage chondrocytes (50). In addition, cartilage-specific deletion of mTOR upregulates autophagy and delays OA (51). Conversely, suppression of regulated in development and DNA damage response 1, an endogenous inhibitor of mTOR, leads to mTOR activation and defective autophagy in OA (52). PPAR $\gamma$ deficiency also results in aberrant mTOR signaling, which induces a metabolic balance in cartilage homeostasis $(24,53)$. It may be hypothesized that activation of autophagy is beneficial to articular cartilage, leading to mTOR suppression and the attenuation of OA $(54,55)$. These findings indicated that Iso may activate autophagy by downregulating the mTORC1 signaling pathway. However, the exact mechanism associated with Iso and the mTORC1 autophagy pathway remains unclear, and it has yet to be established whether autophagy and mTORC1 are direct targets of Iso. Further studies are required to elucidate the underlying mechanism by which Iso regulates the mTORC1 autophagic signaling pathway.

The present study also investigated the in vivo effects of Iso on articular cartilage in a DMM model over a period of 4 weeks. The results revealed that Iso ameliorated the OA-associated effects, and that 4 weeks was sufficient to induce OA using DMM.

The present study had a few limitations. A more in-depth study would provide more information regarding the effects of Iso on cartilage deterioration in DMM. In future studies, the course of DMM-induced OA should be evaluated for 8 weeks. In addition, further insight into the effects of Iso on other aspects of OA is required, including subchondral bone, synovium and meniscus. The incidence of OA increases with age and the treatment options for OA are limited. In the late stages of OA, patients require arthroplasty, which has a large economic burden. Therefore, more attention should be given to developing novel therapies that delay the progression of OA, including exploiting novel drugs from medicinal herbs.

In conclusion, the results of the present study demonstrated that Iso ameliorated articular cartilage degradation in a murine DMM model. A reduction in MMP13, Runx2, Col X and VEGF expression was observed in cartilage from Iso-treated mice following DMM surgery. In addition, autophagy was activated and mTORC1 was inhibited in murine primary chondrocytes. These observations indicated that Iso may ameliorate the pathological alterations induced by OA by delaying chondrocyte deterioration, activating autophagy and inhibiting mTORC1, which suggests that Iso may be an effective therapeutic approach for attenuating articular cartilage degradation and thus, treating OA. 


\section{Acknowledgements}

The present study was partially supported by the Research Fund for the Doctoral Program of Higher Education of China (grant no. 20124433110021).

\section{References}

1. Losina E, Weinstein AM, Reichmann WM, Burbine SA, Solomon DH, Daigle ME, Rome BN, Chen SP, Hunter DJ, Suter LG, et al: Lifetime risk and age at diagnosis of symptomatic knee osteoarthritis in the US. Arthritis Care Res (Hoboken) 65: 703-711, 2013.

2. Musumeci G, Aiello F, Szychlinska M, Di Rosa M, Castrogiovanni P and Mobasheri A: Osteoarthritis in the XXIst century: Risk factors and behaviours that influence disease onset and progression. Int J Mol Sci 16: 6093-6112, 2015.

3. Musumeci G, Szychlinska MA and Mobasheri A: Age-related degeneration of articular cartilage in the pathogenesis of osteoarthritis: Molecular markers of senescent chondrocytes. Histol Histopathol 30: 1-12, 2015.

4. Aigner T, Sachse A, Gebhard PM and Roach HI: Osteoarthritis: Pathobiology-targets and ways for therapeutic intervention. Adv Drug Deliv Rev 58: 128-149, 2006.

5. Jacques C, Gosset M, Berenbaum F and Gabay C: The role of IL-1 and IL-1Ra in joint inflammation and cartilage degradation. Vitam Horm 74: 371-403, 2006.

6. Loeser RF, Goldring SR, Scanzello CR and Goldring MB: Osteoarthritis: A disease of the joint as an organ. Arthritis Rheum 64: 1697-1707, 2012.

7. Yuan Q, Sun L, Li JJ and An CH: Elevated VEGF levels contribute to the pathogenesis of osteoarthritis. BMC Musculoskelet Disord 15: 437, 2014.

8. Musumeci G, Carnazza ML, Loreto C, Leonardi R and Loreto C: $\beta$-Defensin-4 (HBD-4) is expressed in chondrocytes derived from normal and osteoarthritic cartilage encapsulated in PEGDA scaffold. Acta Histochem 114: 805-812, 2012.

9. Musumeci G, Carnazza ML, Leonardi R and Loreto $\mathrm{C}$ Expression of $\beta$-defensin- 4 in 'an in vivo and ex vivo model' of human osteoarthritic knee meniscus. Knee Surg Sports Traumatol Arthrosc 20: 216-222, 2012.

10. Philp AM, Davis ET and Jones SW: Developing anti-inflammatory therapeutics for patients with osteoarthritis. Rheumatology (Oxford) 56: 869-881, 2017

11. Goldring MB and Goldring SR: Osteoarthritis. J Cell Physiol 213: 626-634, 2007.

12. Wang S, Chen Q and He L: Development and validation of a gas chromatography-mass spectrometry method for the determination of isoimperatorin in rat plasma and tissue: Application to the pharmacokinetic and tissue distribution study. J Chromatogr B Analyt Technol Biomed Life Sci 852: 473-478, 2007.

13. Wei Y and Ito Y: Preparative isolation of imperatorin, oxypeucedanin and isoimperatorin from traditional Chinese herb 'bai zhi'Angelica dahurica (Fisch. ex Hoffm) Benth. et Hook using multidimensional high-speed counter-current chromatography. J Chromatogr A 1115: 112-117, 2006.

14. Kang OH, Chae HS, Oh YC, Choi JG, Lee YS, Jang HJ, Kim JH, Kim YC, Sohn DH, Park H and Kwon DY: Anti-nociceptive and anti-inflammatory effects of Angelicae dahuricae radix through inhibition of the expression of inducible nitric oxide synthase and NO production. Am J Chin Med 36: 913-928, 2008.

15. Zheng YM, Shen JZ, Wang Y, Lu AX and Ho WS: Anti-oxidant and anti-cancer activities of Angelica dahurica extract via induction of apoptosis in colon cancer cells. Phytomedicine 23: 1267-1274, 2016.

16. Nie H, Meng LZ, Zhou JY, Fan XF, Luo- Y and Zhang GW: Imperatorin is responsible for the vasodilatation activity of Angelica Dahurica var. Formosana regulated by nitric oxide in an endothelium-dependent manner. Chin J Integr Med 15: 442-447, 2009

17. Chen YF, Tsai HY and Wu TS: Anti-inflammatory and analgesic activities from roots of Angelica pubescens. Planta Med 61: 2-8, 1995.

18. Moon TC, Jin M, Son JK and Chang HW: The effects of isoimperatorin isolated from Angelicae dahuricae on cyclooxygenase-2 and 5-lipoxygenase in mouse bone marrow-derived mast cells. Arch Pharm Res 31: 210-215, 2008.
19. Ban HS, Lim SS, Suzuki K, Jung SH, Lee S, Lee YS, Shin KH and Ohuchi K: Inhibitory effects of furanocoumarins isolated from the roots of Angelica dahurica on prostaglandin E2 production. Planta Med 69: 408-412, 2003

20. Moon L, Ha YM, Jang HJ, Kim HS, Jun MS, Kim YM, Lee YS, Lee DH, Son KH, Kim HJ, et al: Isoimperatorin, cimiside E and 23-O-acetylshengmanol-3-xyloside from Cimicifugae rhizome inhibit TNF- $\alpha$-induced VCAM-1 expression in human endothelial cells: Involvement of PPAR- $\gamma$ upregulation and PI3K, ERK1/2 and, PKC signal pathways. J Ethnopharmacol 133: 336-344, 2011.

21. Glasson SS, Chambers MG, Van Den Berg WB and Little CB: The OARSI histopathology initiative - recommendations for histological assessments of osteoarthritis in the mouse. Osteoarthritis Cartilage 18 (Suppl 3): S17-S23, 2010.

22. Livak KJ and Schmittgen TD: Analysis of relative gene expression data using real-time quantitative PCR and the 2(-Delta Delta C(T)) method. Methods 25: 402-408, 2001.

23. Lotz M and Caramés B: Autophagy: A new therapeutic target in cartilage injury and osteoarthritis. J Am Acad Orthop Surg 20: 261-262, 2012

24. Vasheghani F, Zhang Y, Li YH, Blati M, Fahmi H, Lussier B, Roughley P, Lagares D, Endisha H, Saffar B, et al: PPAR deficiency results in severe, accelerated osteoarthritis associated with aberrant mTOR signalling in the articular cartilage. Ann Rheum Dis 74: 569-578, 2015.

25. Guba M, von Breitenbuch P, Steinbauer M, Koehl G, Flegel S, Hornung M, Bruns CJ, Zuelke C, Farkas S, Anthuber M, et al: Rapamycin inhibits primary and metastatic tumor growth by antiangiogenesis: Involvement of vascular endothelial growth factor. Nat Med 8: 128-135, 2002.

26. Laplante M and Sabatini DM: Regulation of mTORC1 and its impact on gene expression at a glance. J Cell Sci 126: 1713-1719, 2013.

27. Berenbaum F, Griffin TM and Liu-Bryan R: Review: Metabolic regulation of inflammation in osteoarthritis. Arthritis Rheumatol 69: 9-21, 2017.

28. van der Kraan PM and van den Berg WB: Chondrocyte hypertrophy and osteoarthritis: Role in initiation and progression of cartilage degeneration? Osteoarthritis Cartilage 20: 223-232, 2012.

29. Higashikawa A, Saito T, Ikeda T, Kamekura S, Kawamura N, Kan A, Oshima Y, Ohba S, Ogata N, Takeshita K, et al: Identification of the core element responsive to runt-related transcription factor 2 in the promoter of human type $\mathrm{X}$ collagen gene. Arthritis Rheum 60: 166-178, 2009.

30. Kamekura S, Kawasaki Y, Hoshi K, Shimoaka T, Chikuda H, Maruyama Z, Komori T, Sato S, Takeda S, Karsenty G, et al: Contribution of runt-related transcription factor 2 to the pathogenesis of osteoarthritis in mice after induction of knee joint instability. Arthritis Rheum 54: 2462-2470, 2006.

31. Yi JW, Lee WS, Kim SB, Heo YM and Chae DS: Effect of zoledronate on the expression of vascular endothelial growth factor-A by articular chondrocytes and synovial cells: An in vitro study. J Bone Metab 21: 249-255, 2014.

32. Neve A, Cantatore FP, Corrado A, Gaudio A, Ruggieri S and Ribatti D: In vitro and in vivo angiogenic activity of osteoarthritic and osteoporotic osteoblasts is modulated by VEGF and vitamin D3 treatment. Regul Pept 184: 81-84, 2013.

33. Takimoto A, Nishizaki Y, Hiraki Y and Shukunami C: Differential actions of VEGF-A isoforms on perichondrial angiogenesis during endochondral bone formation. Dev Biol 332. 196-211, 2009.

34. Saetan N, Honsawek S, Tanavalee A, Yuktanandana P, Meknavin S, Ngarmukos S, Tanpowpong T and Parkpian V: Relationship of plasma and synovial fluid vascular endothelial growth factor with radiographic severity in primary knee osteoarthritis. Int Orthop 38: 1099-1104, 2014.

35. Kim HR, Lee JH, Kim KW, Kim BM and Lee SH: The relationship between synovial fluid VEGF and serum leptin with ultrasonographic findings in knee osteoarthritis. Int J Rheum Dis 19: 233-240, 2016.

36. Nagai T, Sato M, Kobayashi M, Yokoyama M, Tani Y and Mochida J: Bevacizumab, an anti-vascular endothelial growth factor antibody, inhibits osteoarthritis. Arthritis Res Ther 16: 427, 2014

37. Nagai T, Sato M, Kutsuna T, Kokubo M, Ebihara G, Ohta N and Mochida J: Intravenous administration of anti-vascular endothelial growth factor humanized monoclonal antibody bevacizumab improves articular cartilage repair. Arthritis Res Ther 12: R178, 2010. 
38. Bouderlique T, Vuppalapati KK, Newton PT, Li L, Barenius B and Chagin AS: Targeted deletion of Atg5 in chondrocytes promotes age-related osteoarthritis. Ann Rheum Dis 75: 627-631, 2016.

39. Li YS, Zhang FJ, Zeng C, Luo W, Xiao WF, Gao SG and Lei GH: Autophagy in osteoarthritis. Joint Bone Spine 83: 143-148, 2016.

40. Caramés B, Taniguchi N, Otsuki S, Blanco FJ and Lotz M: Autophagy is a protective mechanism in normal cartilage, and its aging-related loss is linked with cell death and osteoarthritis. Arthritis Rheum 62: 791-801, 2010.

41. Borzì RM, Guidotti S, Minguzzi M, Facchini A, Platano D, Trisolino G, Filardo G, Cetrullo S, D'Adamo S, Stefanelli C, et al: Polyamine delivery as a tool to modulate stem cell differentiation in skeletal tissue engineering. Amino Acids 46: 717-728, 2014

42. Yang RT, Zhang C, Liu Y, Zhou HH and Li ZB: Autophagy prior to chondrocyte cell death during the degeneration of Meckel's cartilage. Anat Rec (Hoboken) 295: 734-741, 2012.

43. Alvarez-García O, García-López E, Loredo V, Gil-Peña H, Rodríguez-Suárez J, Ordóñez FA, Carbajo-Pérez E and Santos F: Rapamycin induces growth retardation by disrupting angiogenesis in the growth plate. Kidney Int 78: 561-568, 2010.

44. Laplante M and Sabatini DM: Regulation of mTORC1and its impact on gene expression at a glance. J Cell Sci 126: 1713-1719, 2013.

45. Howell JJ, Ricoult SJ, Ben-Sahra I and Manning BD: A growing role for mTOR in promoting anabolic metabolism. Biochem Soc Trans 41: 906-912, 2013.

46. Caramés B, Hasegawa A, Taniguchi N, Miyaki S, Blanco FJ and Lotz M: Autophagy activation by rapamycin reduces severity of experimental osteoarthritis. Ann Rheum Dis 71: 575-581, 2012.

47. Bohensky J, Leshinsky S, Srinivas V and Shapiro IM: Chondrocyte autophagy is stimulated by HIF-1 dependent AMPK activation and mTOR suppression. Pediatr Nephrol 25: 633-642, 2010
48. Matsuzaki T, Matsushita T, Tabata Y, Saito T, Matsumoto T, Nagai K, Kuroda R and Kurosaka M: Intra-articular administration of gelatin hydrogels incorporating rapamycin-micelles reduces the development of experimental osteoarthritis in a murine model. Biomaterials 35: 9904-9911, 2014.

49. Takayama K, Kawakami Y,Kobayashi M, Greco N, Cummins JH, Matsushita T, Kuroda R, Kurosaka M, Fu FH and Huard J: Local intra-articular injection of rapamycin delays articular cartilage degeneration in a murine model of osteoarthritis. Arthritis Res Ther 16: 482, 2014.

50. Huang MJ, Wang L, Jin DD, Zhang ZM, Chen TY, Jia CH, Wang Y, Zhen XC, Huang B, Yan B, et al: Enhancement of the synthesis of n-3 PUFAs infat-1 transgenic mice inhibits mTORC1 signalling and delays surgically induced osteoarthritis in comparison with wild-type mice. Ann Rheum Dis 73: 1719-1727, 2014.

51. Zhang Y, Vasheghani F, Li YH, Blati M, Simeone K, Fahmi H, Lussier B, Roughley P, Lagares D, Pelletier JP, et al: Cartilage-specific deletion of mTOR upregulates autophagy and protects mice from osteoarthritis. Ann Rheum Dis 74: 1432-1440, 2015.

52. Alvarez-Garcia O, Olmer M, Akagi R, Akasaki Y, Fisch KM, Shen T, Su AI and Lotz MK: Suppression of REDD1 in osteoarthritis cartilage, a novel mechanism for dysregulated mTOR signaling and defective autophagy. Osteoarthritis Cartilage 24: $1639-1647,2016$

53. Dell'Accio F and Sherwood J: PPAR $\gamma / \mathrm{mTOR}$ signalling: Striking the right balance in cartilage homeostasis. Ann Rheum Dis 74: 477-479, 2015.

54. Chagin AS: Effectors of mTOR-autophagy pathway: Targeting cancer, affecting the skeleton. Curr Opin Pharmacol 28: 1-7, 2016.

55. Shi J, Zhang C, Yi Z and Lan C: Explore the variation of MMP3, JNK, p38 MAPKs, and autophagy at the early stage of osteoarthritis. IUBMB Life 68: 293-302, 2016. 